\title{
HIGH-RESOLUTION RADIO OBSERVATIONS OF FIVE PLANETARY NEBULAE
}

\author{
B. ELSMORE \\ (Mullard Radio Astronomy Observatory, Cambridge, England)
}

\section{ABSTRACT}

Observations have been made of 5 planetary nebulae at 1407 and $408 \mathrm{MHz}$ with beam widths of $23^{\prime \prime}$ of arc and $80^{\prime \prime}$ of arc respectively. Measurements of positions, fluxes and angular structure have been obtained; some results are presented in the form of contour maps.

The Cambridge 1-mile radio telescope has been used to map the structure of 5 planetary nebulae. The radio telescope and its mode of operation have been described elsewhere (Ryle, 1962; Elsmore et al., 1966), and only a brief account is given here.

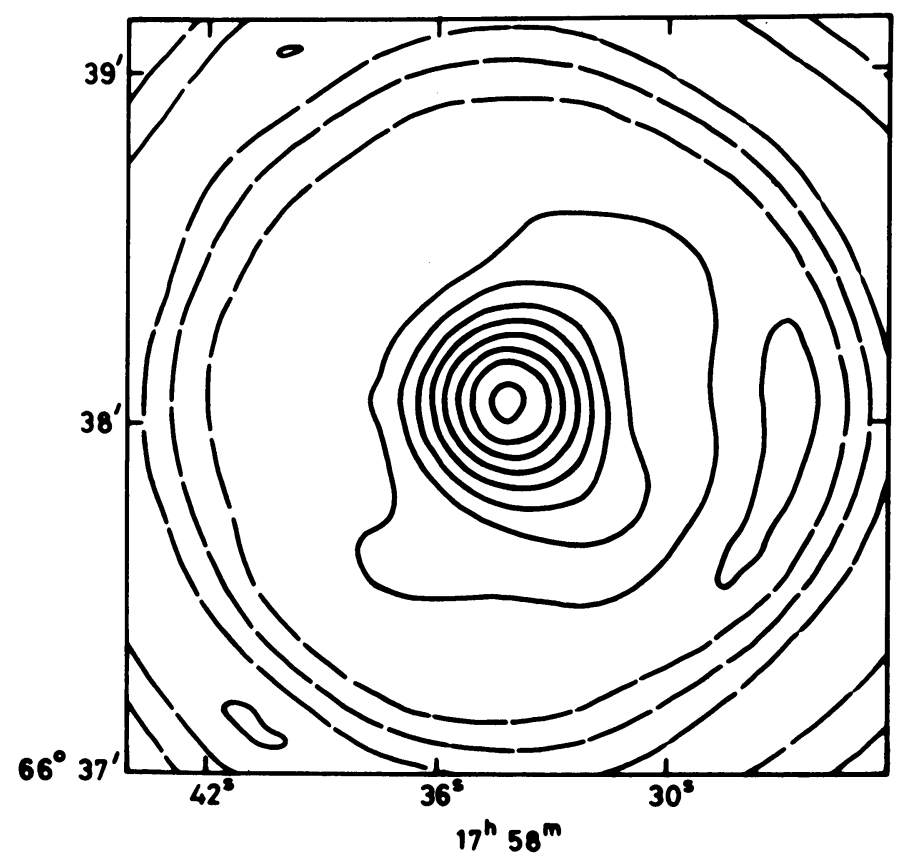

Fig. 1. $1407 \mathrm{MHz}$ map of NGC 6543. (The broken contours are due to the grating side-lobe response of the instrument.)

Osterbrock and O'Dell (eds.), Planetary Nebulae, 108-111. (C. I.A.U. 


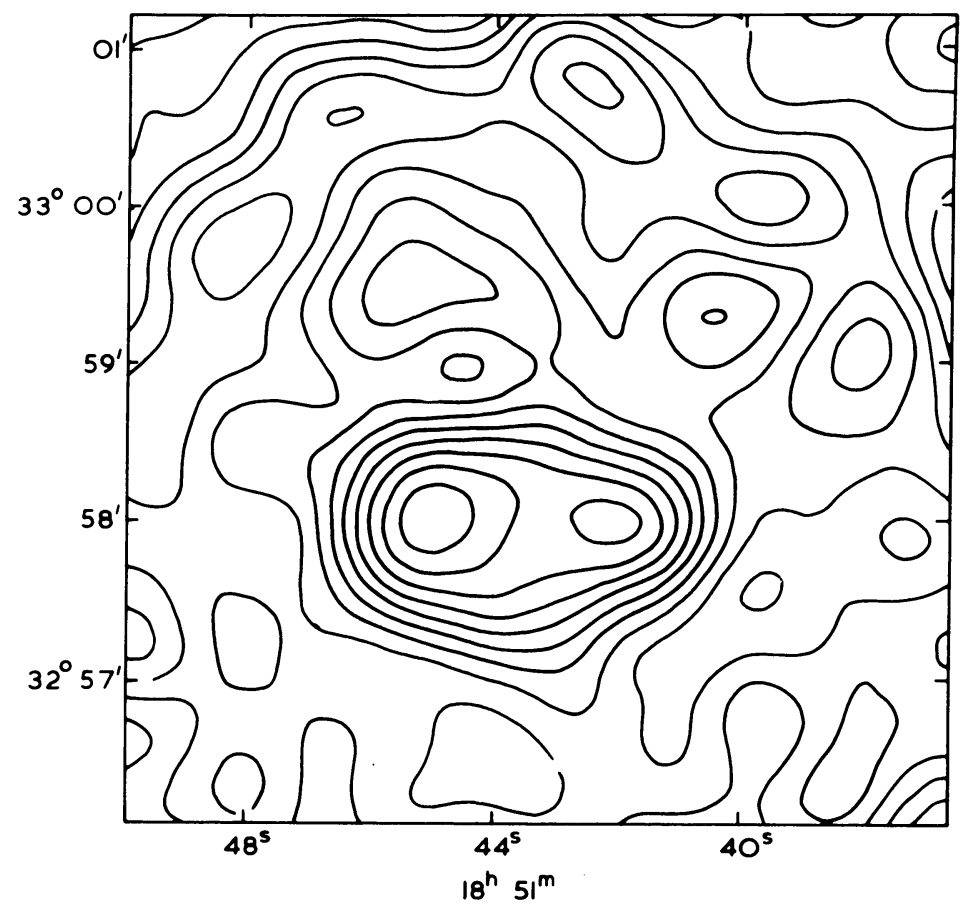

FIG. 2. $1407 \mathrm{MHz}$ map of NGC 6720. The distance between the two peaks of radio emission is $36^{\prime \prime}$ of arc.

Each nebula was observed continuously for at least one 12-hour period using aerials mounted on an East-West base line. The rotation of the Earth carries one aerial around the other, enabling the amplitude and phase of the radiation from the nebula to be sampled along a circular strip. The radius of this strip is equal to the distance between the aerials.

If observations of the nebula are combined with those made at different spacings, a pencil-beam response is obtained in the vicinity of the nebula, together with a circular-grating response, the angular separation of which depends upon the number of different spacings used. Observations were made at two frequencies simultaneously, $1407 \mathrm{MHz}$ and $408 \mathrm{MHz}$, giving pencil-beam widths of $23^{\prime \prime}$ of arc and $80^{\prime \prime}$ of arc in right ascension; the beam widths being greater in declination by a factor $\operatorname{cosec} \delta$.

The results appear in the form of contour maps of radio intensity of which four are shown in Figures 1-4. The declination scale of the maps has been compressed by a factor sine $\delta$ so that the beam appears circular. A summary of the observations is given in Table 1.

Generally, the radio emission originates in a region slightly smaller than that of the optical nebula except in the case of NGC 7662, where the size of the nebula at 1407 


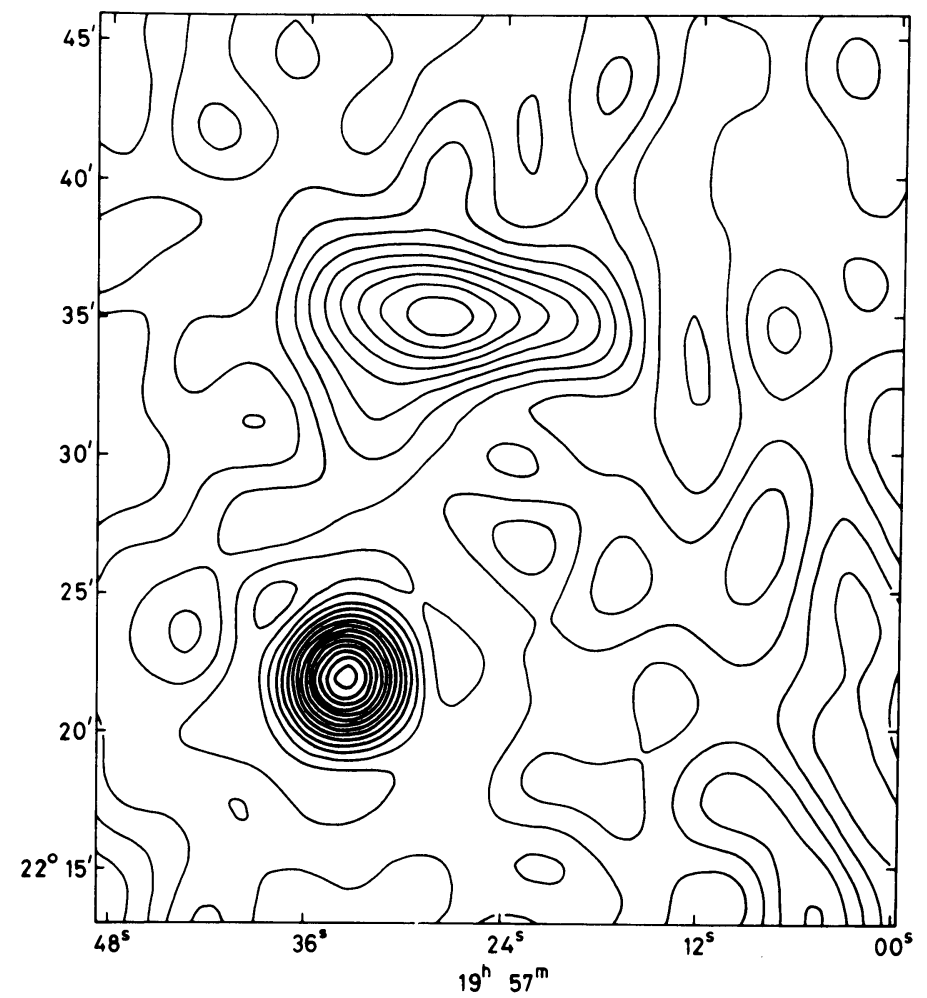

Fig. 3. $408 \mathrm{MHz}$ map of NGC 6853. The object shown at declination $22^{\circ} 22^{\prime}$ is a small diameter source of 0.5 flux units, which is not related to the nebula.

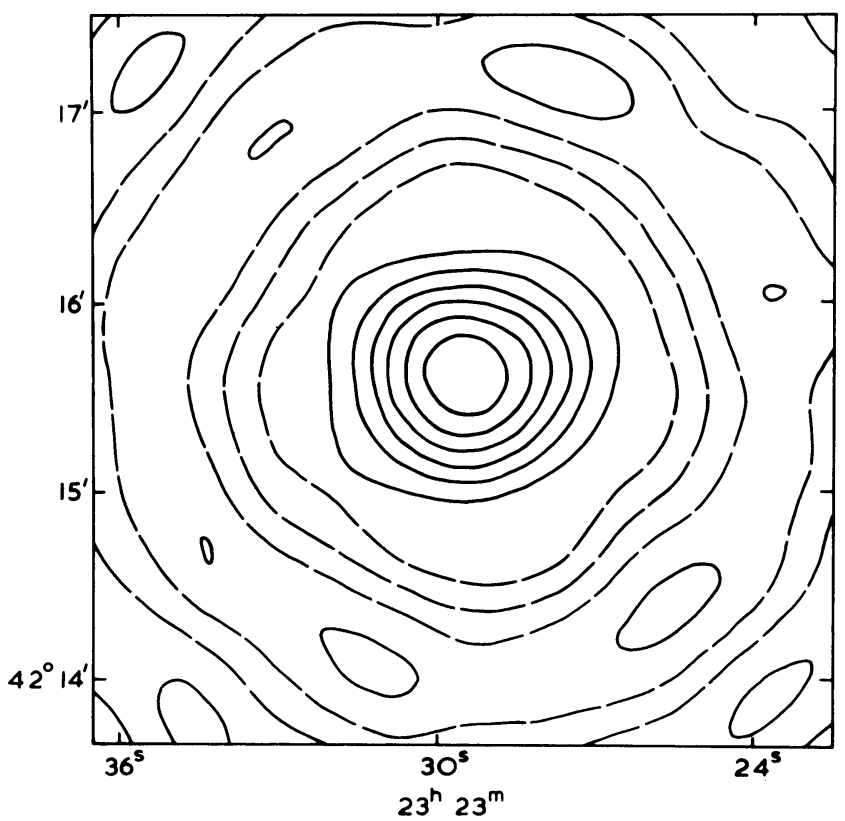

Fig. 4. $1407 \mathrm{MHz}$ map of NGC 7662. (The broken contours are due to the grating side-lobe response of the instrument.) 
Table 1

$\begin{array}{lccc}\text { NGC } & \begin{array}{c}\text { Integrated Flux } \\ 1407 \mathrm{MHz}\end{array} & \begin{array}{c}0^{-26} \mathrm{Wm}^{-2} \mathrm{~Hz}^{-1} \\ 408 \mathrm{MHz}\end{array} & \text { Width to Half Power at } 1407 \mathrm{MHz} \\ 6543 & 0 \cdot 7 & \leqslant 0 \cdot 2 & <10^{\prime \prime} \\ 6720 & 0.45 & 0.15 & \mathrm{EW}=62^{\prime \prime} \mathrm{NS}=47^{\prime \prime} \\ 6853 & 0.9(+0.3) & 1 \cdot 2 & - \\ 7027 & 1 \cdot 3 & \leqslant 0 \cdot 2 & \left(\text { At } 408 \mathrm{MHz}: \mathrm{EW}=190^{\prime \prime}, \mathrm{NS}=140^{\prime \prime}\right) \\ 7662 & 0.51 & 0.25 & \mathrm{EW}=28^{\prime \prime} \mathrm{NS}=36^{\prime \prime}\end{array}$

$\mathrm{MHz}$ is slightly larger than the optical size. In all cases the centre of the radio emission coincides closely with the centre of the visible nebula.

The fluxes given in Table 1 are believed to be accurate to $\pm 10 \%$ and are in general agreement with previous observations, with the exception of the measurement of NGC 6720 at $408 \mathrm{MHz}$, which disagrees with the value $0 \cdot 5 \pm 0 \cdot 12 \mathrm{f}$.u. measured at $430 \mathrm{MHz}$ by Terzian (1966). There is no evidence for non-thermal emission.

\section{References}

Elsmore, B., Kenderdine, S., Ryle, M. (1966) Mon. Not. R. astr. Soc., 134, 87.

Ryle, M. (1962) Nature, 194, 517.

Terzian, Y. (1966) Astrophys. J., 144, 657.

\section{DISCUSSION}

Kaftan-Kassim: I would like to ask why NGC 7293, the largest planetary nebula, was not observed. I have attempted to map it at $2 \mathrm{~cm}$ using the $140-\mathrm{ft}$ dish at Green Bank with a $2^{\prime}$ beam. A double structure similar to that shown for NGC 6720 was observed.

Elsmore: NGC 7293 is too far South to be observed by the Cambridge 1-mile telescope.

Sheglov: Does the radio centre of NGC 6853 coincide with the central star of this nebula or with the brightest part of the planetary?

Elsmore: The peak of the radio emission coincides closely with the centre of the nebula rather than with the optically brightest region. 